\title{
Handheld surface-enhanced Raman scattering identification of dye chemical composition in felt-tip pen drawings
}

\author{
Daniela Saviello ${ }^{1}$ | Alexandra Di Gioia ${ }^{1}$ | Pierre-Ives Turenne ${ }^{1}$ | Maddalena Trabace $^{2}$ | \\ Rodorico Giorgi $^{2}$ (D) । Antonio Mirabile ${ }^{3}$ (D) | Piero Baglioni ${ }^{2}$ | Daniela Iacopino ${ }^{1}$ (D)
}

\begin{abstract}
${ }^{1}$ Tyndall National Institute, Cork, Ireland
${ }^{2}$ Department of Chemistry and CSGI, University of Florence, Sesto Fiorentino, Italy

${ }^{3}$ Mirabile, Paris, France

\section{Correspondence}

Daniela Iacopino, Tyndall National Institute, Dyke Parade, Cork, Ireland. Email: daniela.iacopino@tyndall.ie

Funding information

H2020 NMBP, Grant/Award Number: 646063; Irish Research Council (IRC), Grant/Award Number: GOIPD/2015/716; European H2020 Framework Programme, Grant/Award Number: 646063
\end{abstract}

\begin{abstract}
A handheld Raman spectrometer is used for the first time to perform surfaceenhanced Raman scattering (SERS) analysis of felt-tip pen drawings on paper. The use of SERS combined with near-infrared excitation wavelength allows overcoming the strong fluorescence background interference so that diagnostic spectra are obtained from drawings made by 18 pens of 10 different colors and five different brands. The analysis reveals that often mixtures of two or more dyes are used by pen manufactures in order to obtain the desired color hue and also that same colors from different pen brands often display different dye composition. Handheld SERS is also used for revealing the dye content of historical felt tip pens extensively used by the Italian film director Federico Fellini. The results from this work open the way to the use of handheld instrumentation for analysis of ink-based art works and documents and also provide a starting point towards the creation of a reference SERS database for dye identification.
\end{abstract}

\section{KEYWORDS}

drawing, Federico Fellini, felt-tip pens, handheld Raman, SERS

\section{1 | INTRODUCTION}

Since their introduction in the market in 1953, felt-tip pens have become ubiquitous, being used for a wide range of purposes including educational, professional, commercial and classification/cataloguing. Interestingly, because of their low cost, combined with widespread commercial availability and large variety of color hues, felt-tip pens have also been adopted by many artists as preferred production medium. Consequently, a large cultural heritage of ink-based documents and drawings is today hosted by many modern art museums and galleries around the world. In contrast with organic pigments of natural origin constituting classical artworks, the colorant components of felt-tip pens are industrially produced artificial dyes. The high chemical lability (water solubility and easy photo-degradation) of such dyes and their general incompatibility to classical solvent conservation treatments makes modern artworks easily prone to damage and degradation and poses great challenges to their conservation. ${ }^{[1]}$ Because of the different physicochemical properties of artificial dyes compared with pigments, the long term preservation of modern inkbased artworks requires the development of tailored conservation protocols, which in turn requires knowledge of the chemical dye content in commercial ink mixtures. At present, the acquisition of this information is complicated by the complex composition of felt-tip pens, 
comprising a mixture of organic colorants, solvents, additives, and stabilisers. ${ }^{[2]}$ Additional challenges include the fragmented nature of information available, limited by trademark protection, the variation of chemical composition between brands and year of production, and the absence of a database of information. Last but not least, the design of conservation protocols tailored to modern artworks would benefit from the availability of sensitive analytical techniques allowing in situ characterization in order to (a) investigate the behavior of inks on relevant matrices, (b) test the impact of novel designed conservation treatments, and (c) anticipate precisely the reaction to conservation treatments.

A number of optical techniques mainly based on Fourier transform infrared spectroscopy (FTIR) and Raman spectroscopy have been applied to the characterization of inks and pigments in artworks. ${ }^{[3-5]}$ However, such methods are generally not suitable to the analysis in situ (i.e., no extraction) of the small concentrations of fugitive dyes present in modern paper-based artistic productions. In fact, on one hand, long acquisition times or high laser powers or relatively large (mg) amount of sample would be required in order to obtain acceptable signal/ noise level by FTIR. On the other hand, the large fluorescence interference from both dyes and paper substrates would obscure diagnostic Raman signals. Recently, Sodo et al. applied Raman spectroscopy to investigate the dye content of commercial felt-tip pen drawings. ${ }^{[6]}$ Although some information was achieved, strong background signals from the paper required the complementary use of chromatography techniques to reach reliable conclusions on the chemical composition of the analyzed drawings.

In recent years, these limitations were overcome by the use of surface-enhanced Raman scattering (SERS), a phenomenon associated with the enhancement of Raman signals (up to a factor $10^{6}-10^{8}$ ) experienced by an analyte into close proximity to a roughened noble metal surface. ${ }^{[7]}$ The SERS phenomenon arises mainly from the generation of intensified local electromagnetic fields resulting from localized surface plasmon resonances (LSPR) of the nanoscale surfaces (electromagnetic effect $[\mathrm{EM}])^{[8,9]}$ However, additional charge transfer effects (chemical effects $[\mathrm{CM}]$ ) between the analyte and the nanoscale surface can also contribute to the final observed enhancement. ${ }^{[9]}$ Recently, the applicability of SERS for identification of natural organic colorants used in paints, glazes, and textiles on a variety of works of art was demonstrated. ${ }^{[10-12]}$ SERS was also used by our group and others for the identification of dye content in ballpoint pens. ${ }^{[13-16]}$ Recently, SERS was also used in combination with other analytical techniques for the identification of dyes in commercial felt-tip pens ${ }^{[17,18]}$ and used to identify colorants in colored inks used by artists such as Lucio Fontana and Federico Fellini. ${ }^{[19,20]}$ As an increasing number of modern artworks are already entering a state of high degradation, the inherent noninvasiveness, high sensitivity, and efficient quenching of interfering fluorescence signals could make SERS the analytical technique of choice to support the development of long-term conservation methods for modern artworks.

In this work, we present for the first time the use of Raman handheld instrumentation for the elucidation of dye composition in commercial felt-tip pen ink mixtures. Colored squared areas were drawn on commercial paper with felt-tip pens. Drawings were analyzed in situ by normal Raman (NR) and SERS solely by the use of a handheld Raman spectrograph with excitation wavelength at $785 \mathrm{~nm}$. Such wavelength was selected as it was shown to minimize fluorescence interference from binding media and most of the organic dyes in art applications. ${ }^{[21]}$ In order to prove the wide applicability of the method, nine colors and five marker brands were purchased and analyzed. In the case of red colors, NR was effective in obtaining diagnostic spectra and was used for chemical identification. However, in all other cases, NR signals were obscured from interference fluorescence and only SERS gave successful chemical identification. The analysis revealed that in some cases, same color pens of different brands displayed different dye compositions and that often a blend of dyes was used to obtain the desired color hue. Handheld SERS was also used for the identification of dye composition in felt-tip pens belonging to the late Italian film director Federico Fellini. These data suggest that SERS with excitation in the near-infrared can be applied to sensitive analysis of modern pens. The effective suppression of fluorescence interference allowed the use of light-weight handheld Raman instrumentation, which has great implications for analysis of unmovable and irregularly shaped art objects. Additionally, the data collected from this work constitute an important step in the development of SERS spectral libraries for dye identification, which would nicely complement corresponding databases already available for natural colorants used in art applications. ${ }^{[22]}$

\section{2 | RESULTS AND DISCUSSION}

Ten felt-tip pens from the popular commercial Stabilo brand were selected for this work (see Table 1 for a list of all analyzed pens). The colors ranged from red and pink to blue and green.

Ag nanoinks used as SERS probes were synthesized following the synthesis reported by Polavarapu et al. ${ }^{[23]}$ The method started with the synthesis of high volume 
TABLE 1 List of all analyzed Stabil o felt tip pens

\begin{tabular}{lll}
\hline Brand & Name & Color \\
\hline Stabilo & R_68-48 & Red \\
\hline Stabilo & W_68-19 & Wine \\
\hline Stabilo & P_68-056 & Pink \\
\hline Stabilo & M_68-56 & Magenta \\
\hline Stabilo & N_68-22 & Navy \\
\hline Stabilo & G_68-36 & Green \\
\hline Stabilo & L_68-58 & Lilac \\
\hline Stabilo & PR_68-55 & Purple \\
\hline Stabilo & LB_68-41 & Light blue \\
\hline Stabilo & C_68-31 & Cyan \\
\hline
\end{tabular}

Ag nanoparticle solutions, which were subsequently concentrated up to 100 times in order to obtain highly viscous solutions. The characterization of Ag nanoinks was carried out by Scanning electron microscopy (SEM) and UV-vis spectroscopy and showed formation of particles mostly spherical in shape, with an average size of $100 \pm 5 \mathrm{~nm}$ and a plasmonic peak centered at $416 \mathrm{~nm}$ (see Figure S1). Such ink-like suspensions were inserted in commercial fountain pen cartridges and deposited on analytical areas by a so called pen-on-paper (POP) method, which consisted in pen writing of $\mathrm{Ag}$ nanoink features on paper areas colored by felt-tip pens. ${ }^{[23]}$ The POP approach was essential to obtain reproducible SERS features for reliable Raman portable instrumentation analysis directly on colored paper. In fact, the felt-tip pens analyzed in this work were highly water soluble and direct deposition (by syringe or Gilson pipette) of $\mathrm{Ag}$ nanoink aqueous suspension on colored paper caused dissolution of the pen and discoloration of the SERS adjacent area (Figure 1a,b). This in turn drastically decreased the concentration of the dye in contact with the $\mathrm{Ag}$ nanoink and made impossible the recording of SERS spectra. In contrast, POP deposition of $\mathrm{Ag}$ nanoinks on colored paper allowed to minimize the amount of solvent deposited on paper, ensured precise and controlled nanoink deposition, and generated size-, shape-, and location-customized SERS active areas (Figure 1c,d). Furthermore, POP deposition was essential to obtain reliable SERS spectra by portable instrumentation whereby a microscope was not used to locate the impinging position of the laser, and SERS detection was greatly facilitated by the controlled deposition of relatively large (millimeter size) SERS-active areas.
FIGURE 1 Photographs of (a) Ag nanoink suspension, (b) Stabilo R_68-48 colored paper square with drop deposited Ag nanoink, (c) fountain pen containing Ag nanoinks, (d) Stabilo R_6848 colored square paper and "NANOINK" written by pen on paper approach
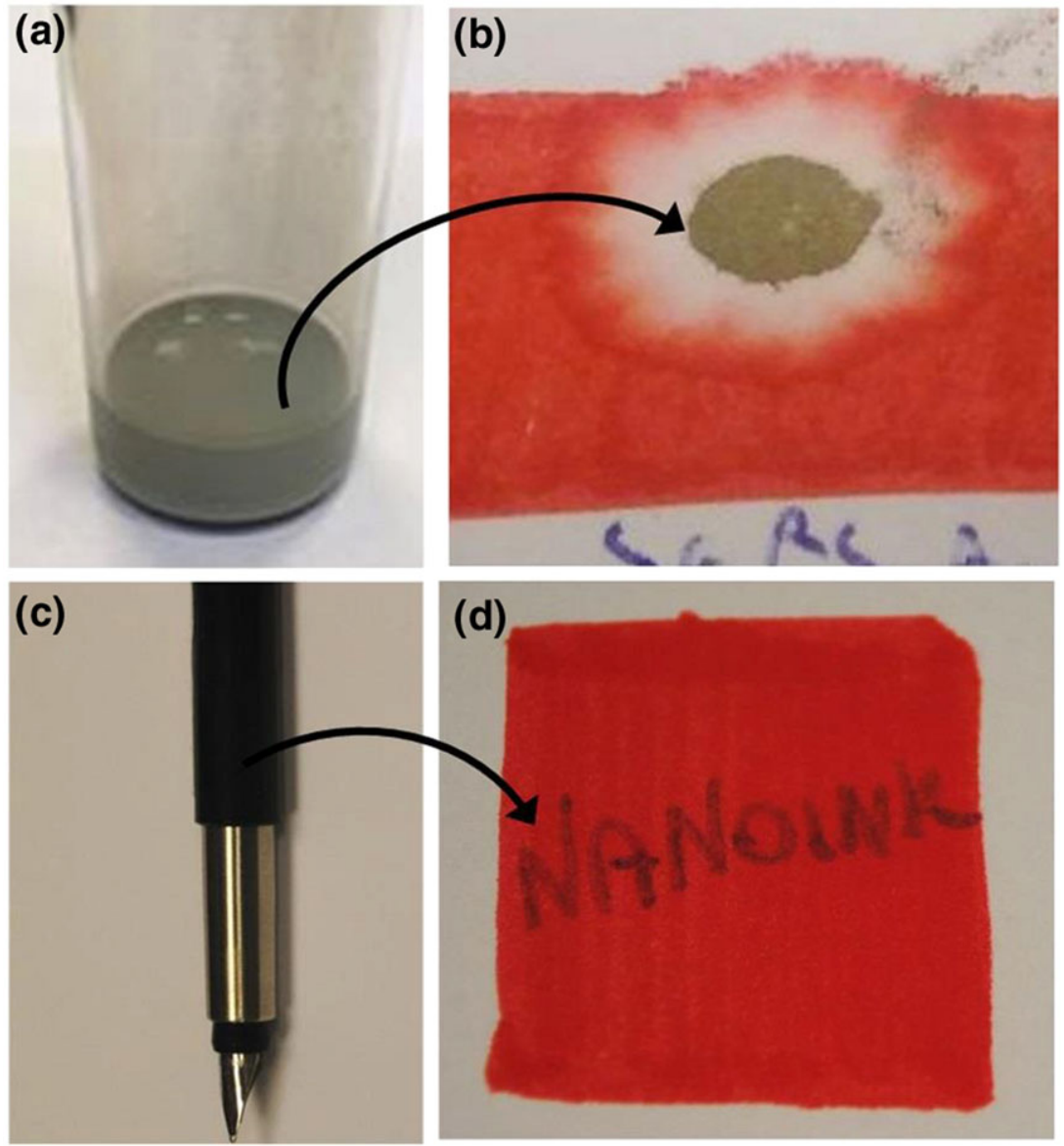
Figure 2 shows the NR and SERS spectra of six selected colored areas (red, wine, pink, magenta, navy, and green; four additional colors are shown in Figure S2) directly measured on paper substrates. For each spectrum, also, the SERS spectrum of Ag nanoinks deposited on paper is shown (SERS blank), to highlight the SERS response of the uncolored paper substrate. For all colors (with the exception of red R_68-48, Figure 2a) the NR spectra measured with handheld instrumentation at illumination wavelength $785 \mathrm{~nm}$ were saturated or showed low intensity peaks, mostly attributable to the paper substrate. In contrast, SERS spectra measured under the same experimental conditions showed higher intensity and diagnostic peaks. The position of the peaks differed from sample to sample and also from the blank, further demonstrating that the achieved spectra were diagnostic of the analyzed pen colors.

The data of Figure 2 showed that SERS was (in general) effective in enhancing the Raman response of felttip pens and could be therefore used for dye identification in pen color mixtures. In order to prove this point, the spectra of Figure 2b-f were baseline subtracted and plotted against the SERS spectra of red, blue, and yellow reference dyes. The spectrum of the wine color W_68-19 (Figure 3a) showed peaks at $1626 \mathrm{~cm}^{-1}$ (aromatic C-C bending and $\mathrm{C}=\mathrm{C}$ stretching), $1506 \mathrm{~cm}^{-1}$ (aromatic
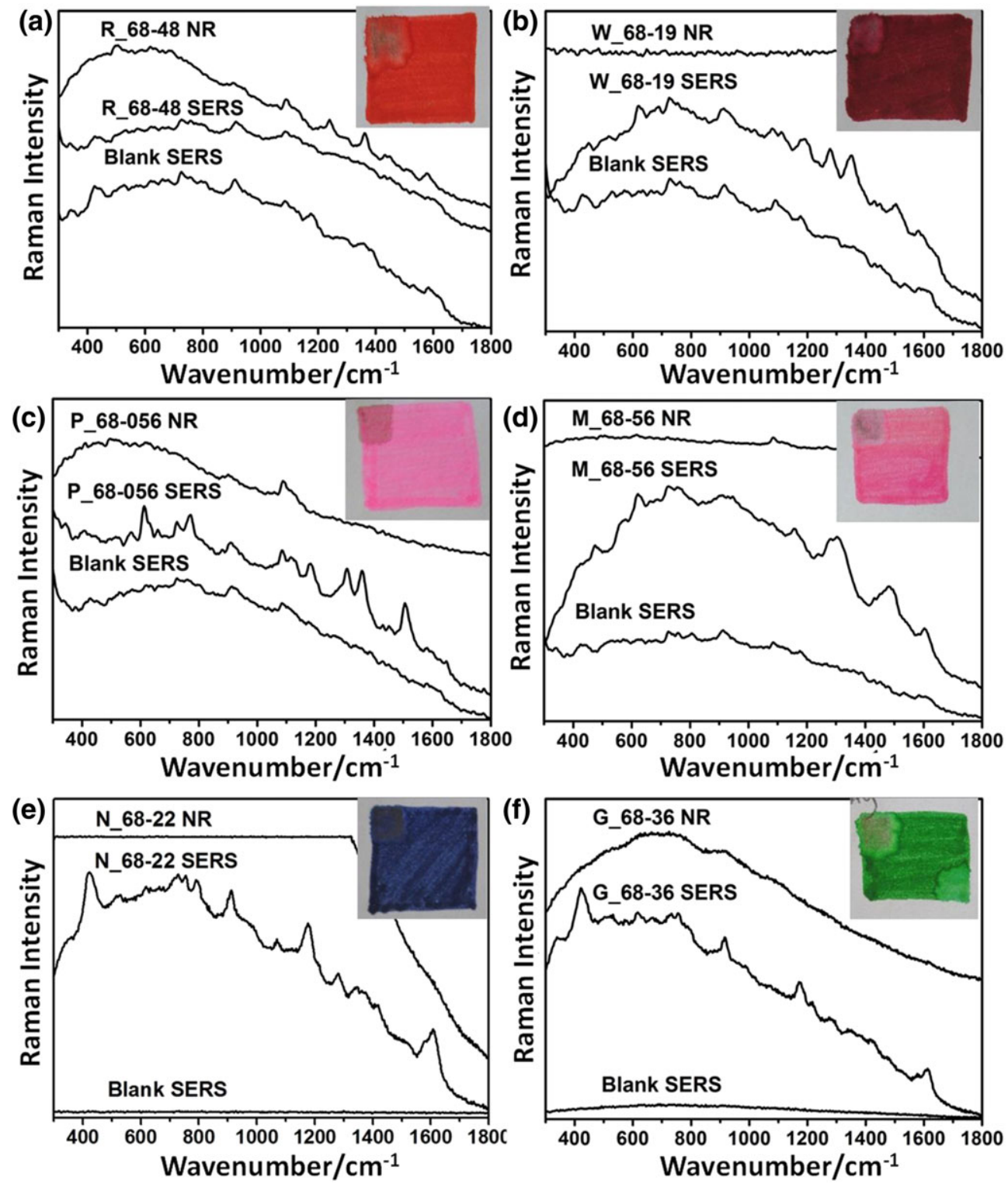

FIGURE 2 normal Raman (NR), surface-enhanced Raman scattering (SERS), and blank SERS spectra recorded at $785 \mathrm{~nm}$ for colored squares on paper drawn by Stabilo felt-tip pens: (a) red square R_68-48, acquisition time $10 \mathrm{~s}$, (b) wine square, A_68-19, acquisition time $10 \mathrm{~s}$, (c) pink square, P_68-056, acquisition time $7.41 \mathrm{~s}$, (d) magenta square, M_68-56, acquisition time 7.41 s, (e) navy square, N_68-22, acquisition time $0.31 \mathrm{~s}$, (f) green square, G_68-36, acquisition time $0.5 \mathrm{~s}$. For each spectrum a blank SERS spectrum was recorded under the same acquisition time of NR and SERS spectra. Inset: Photographs of analyzed colored paper squares 

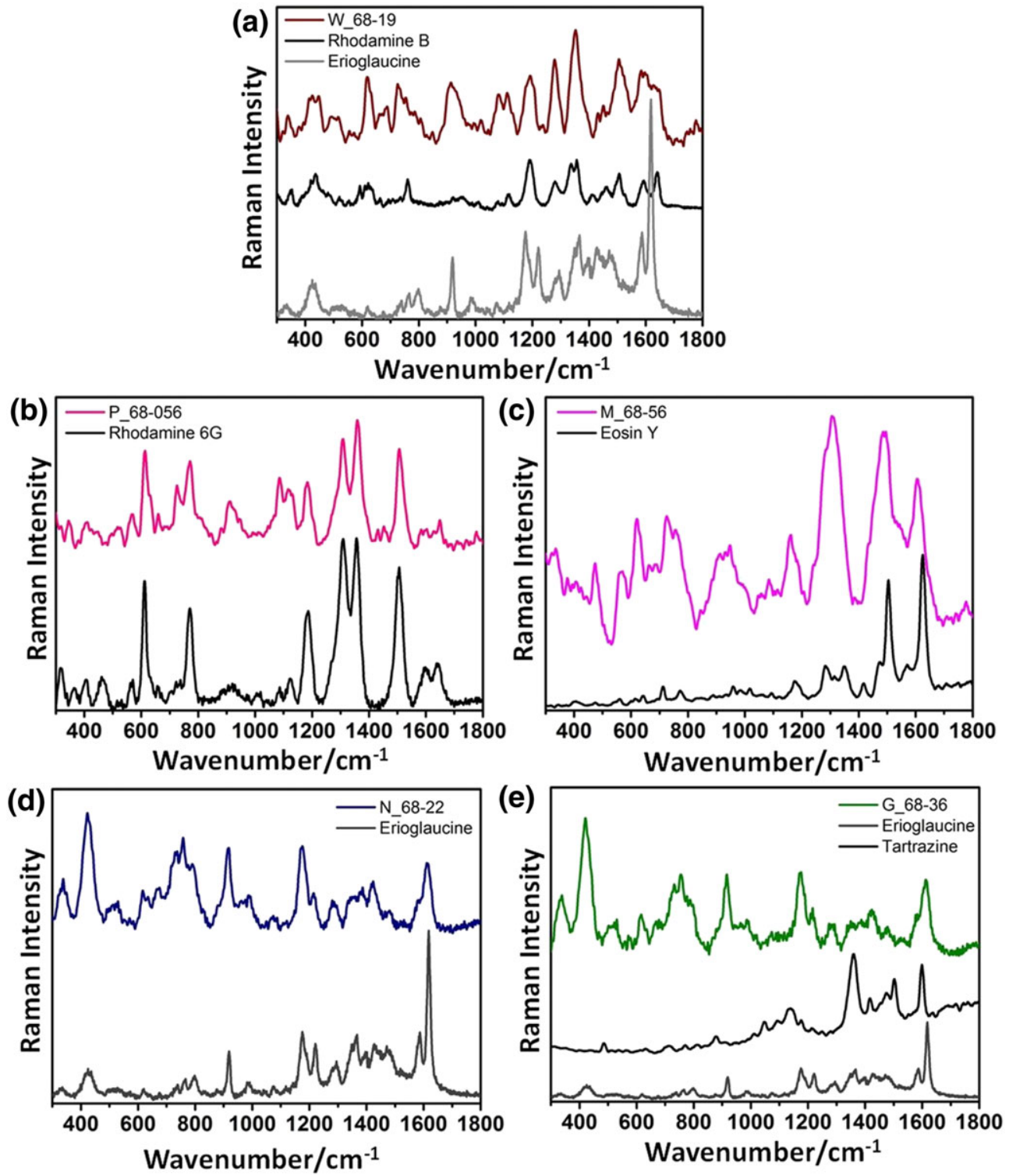

FIGURE 3 Identification of felt-tip pens dye composition. Surface-enhanced Raman scattering spectra of Stabilo colored squares on paper against surface-enhanced Raman scattering spectra of references: (a) wine square W_68-19 pen, erioglaucine, and rhodamine B references; (b) pink square P_68-056 pen and rhodamine 6G reference; (c) magenta square M_68-56 pen and pink eosin Y reference; (d) navy square N_62-22 pen and erioglaucine reference; (e) green square G_68-36 pen, erioglaucine, and tartrazine references

$\mathrm{C}-\mathrm{H}$ bending), $1277 \mathrm{~cm}^{-1}$ (aromatic $\mathrm{C}-\mathrm{C}$ bending), $1193 \mathrm{~cm}^{-1}(\mathrm{C}-\mathrm{C}$ bridge band stretching and aromatic $\mathrm{C}-\mathrm{H}$ bending) diagnostic of red rhodamine $\mathrm{B}$ dye $\mathrm{e}^{[24]}$ and peaks at $1587 \mathrm{~cm}^{-1}$ (aromatic $\mathrm{C}-\mathrm{C}$ stretching), and $914 \mathrm{~cm}^{-1}$ (C-C ring stretching) diagnostic of the blue dye erioglaucine. ${ }^{[25]}$ The spectrum of pink P_68-056 (Figure 3b) showed good overlap with the spectrum of rhodamine 6G. Specifically, peaks at $1503 \mathrm{~cm}^{-1}(\mathrm{C}-\mathrm{N}$ stretching, $\mathrm{C}-\mathrm{H}$ bending, $\mathrm{N}-\mathrm{H}$ bending), $1358 \mathrm{~cm}^{-1}$ (C-H bending, $1306 \mathrm{~N}-\mathrm{H}$ bending, $\mathrm{CH}_{2}$ wagging), 769 and $614 \mathrm{~cm}^{-1}(\mathrm{C}-\mathrm{H}$ bending) overlapped with corresponding peaks of rhodamine $6 \mathrm{G}$. Interestingly, although similar in color to pink, magenta M_68-56 showed chemical composition different than P_68-056. In fact
(Figure 3c), peaks at $1626 \mathrm{~cm}^{-1}$ (xanthene ring $\mathrm{C}-\mathrm{C}$ stretching) and $1506 \mathrm{~cm}^{-1}$ (xanthene and benzene ring $\mathrm{C}-\mathrm{C}$ stretching) diagnostic of the presence of eosin Y were found for this color felt-tip pen. ${ }^{[21,26]}$ Navy N_68-22 color (Figure 3d) showed diagnostic peaks of erioglaucine. Specifically, vibrational bands were observed at 1618-1587 $\mathrm{cm}^{-1}$ (aromatic C-C stretching), 1481$1452 \mathrm{~cm}^{-1}$ (ring deformation), $1420-1370 \mathrm{~cm}^{-1}(\mathrm{~N}-\mathrm{C}$ aromatic stretching), $1279 \mathrm{~cm}^{-1}$ (aromatic $\mathrm{C}-\mathrm{C}$ stretching), and $1185 \mathrm{~cm}^{-1}$ (in-plane ring $\mathrm{CH}$ bending). Finally (Figure 3e), green G_38-36 was a mixture of blue erioglaucine (1618 and $1185 \mathrm{~cm}^{-1}$ peaks) and yellow tartrazine $\left(1505 \mathrm{~cm}^{-1}, \mathrm{C}=\mathrm{C}\right.$ pyrazole bending, $1364 \mathrm{~cm}^{-1}$, azo group stretching and symmetrical 

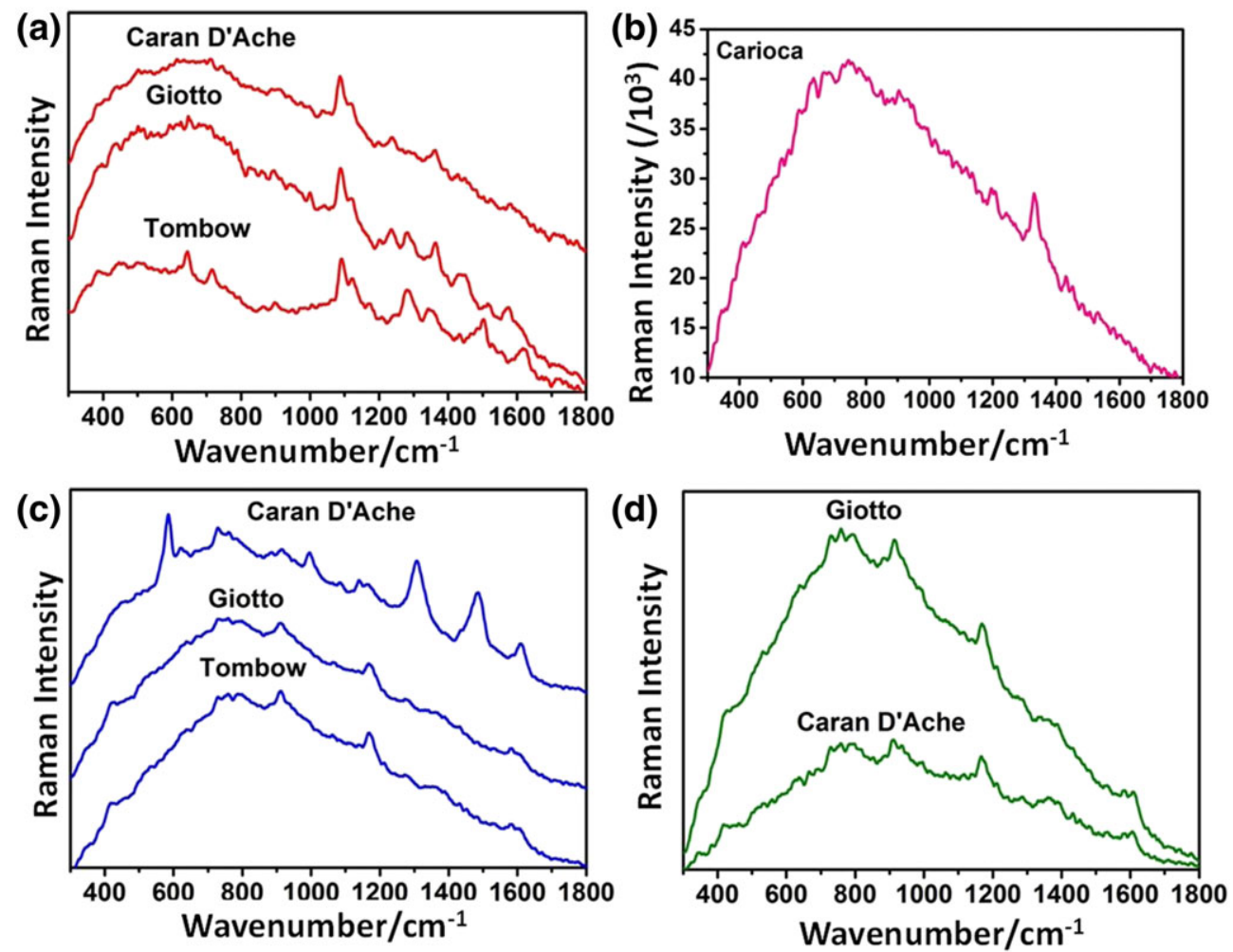

FIGURE 4 (a) Normal Raman spectra of red Caran D'Ache, Giotto, and Tombow colored paper squares; (b) surface-enhanced Raman scattering (SERS) spectra of pink carioca doodles colored paper squares; (c) SERS spectra of blue Caran D'Ache, Giotto, and Tombow colored paper squares; (d) SERS spectra of green Caran D'Ache and Giotto colored paper squares
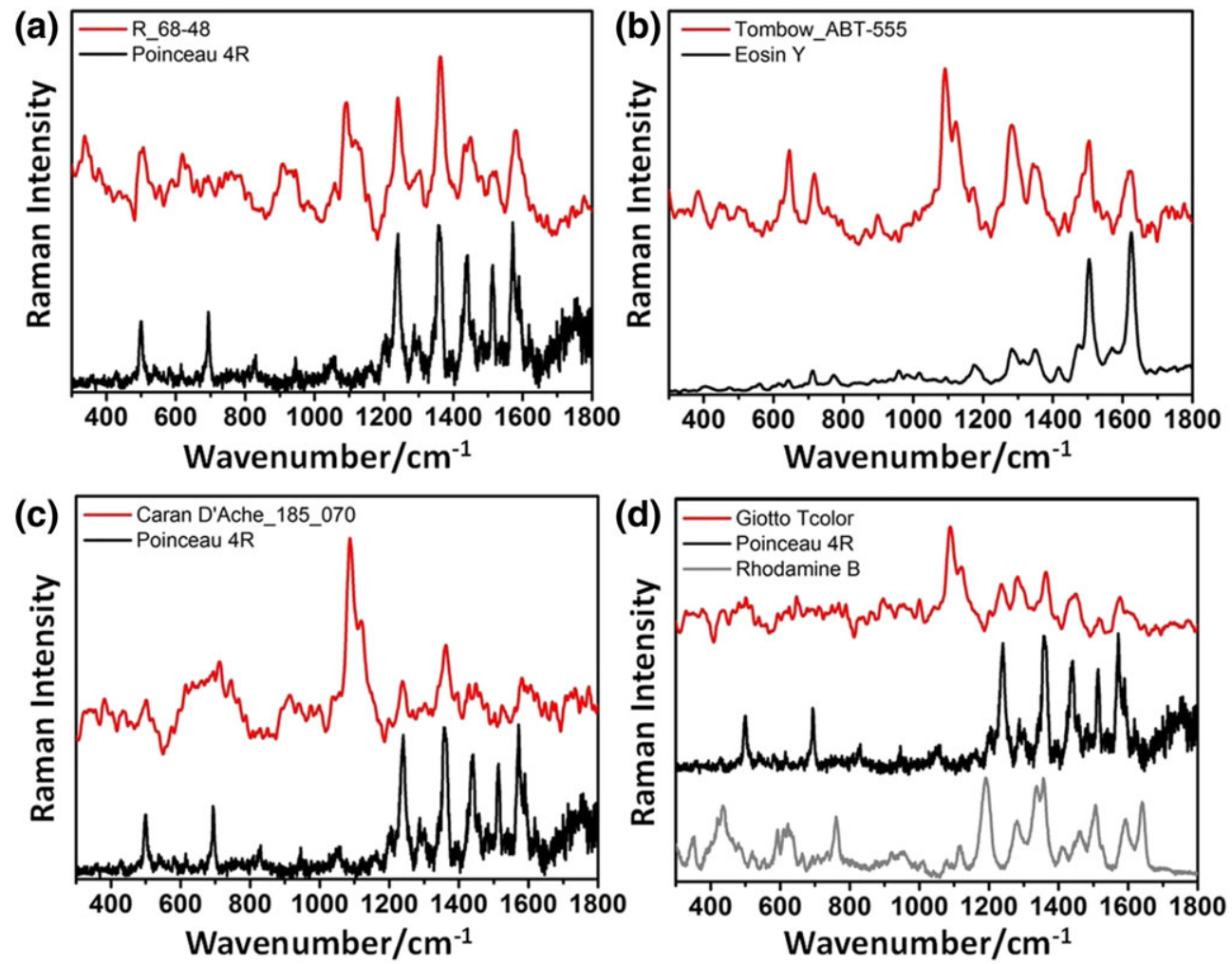

FIGURE 5 Identification of pen dye composition. Surface-enhanced Raman scattering spectra of red colored squares drawn by different red color brands against surface-enhanced Raman scattering spectra of references: (a) Stabilo 68-48 square and poinceau 4R, (b) Tombow ABT-555 square and eosin Y, (c) Caran D‘Ache 185-070 square and poinceau 4R, (d) Giotto_Tcolor square, poinceau 4R, and rhodamine B 
stretching of the carboxyl group) dyes. ${ }^{[27]}$ Regarding the additional analyzed colors (Figure S2), no diagnostic peaks were obtained for the lilac color L_68-58, whereas purple, light blue, and cyan colors showed the same spectral features as navy N_68-22 and were therefore considered to be constituted mainly by erioglaucine.

In order to show wider applicability, the analysis was extended to other pen brands. Specifically, Figure 4 shows the result of NR and SERS analysis performed on selected colors of Caran D'Ache, Giotto, Tombow, and Carioca pens. Interestingly, red Caran D'Ache, Giotto, Tombow (Figure 4a) showed the same behavior as the red Stabilo R_68-48 of Figure 1a, showing more diagnostic NR spectra than SERS spectra. For pink Carioca, blue (Caran D'Ache, Giotto, and Tombow) and green (Caran D'Ache and Giotto) colors SERS spectra were more intense and diagnostic than NR spectra. Spectra of different colors showed different spectral features, again showing evidence of different chemical composition. Interestingly, also among the same color, red and blue pens showed different peaks, indicative of different chemical compositions used by different brands.

In order to better illustrate this point, Figure 5 show SERS spectra of the four analyzed red colors (Stabilo, Caran D'Ache, Giotto, and Tombow) plotted together with red reference dyes. Dye poinceau $4 \mathrm{R}$ constituted the main component of reds Stabilo R_68-48, Caran D'Ache 185-070 and Giotto Tcolor (Figure 5a,c,d). Specifically, peaks at 1238,1362,1436,1517, and $1580 \mathrm{~cm}$ ${ }^{-1}$ were found in red Stabilo and Giotto pens and reference poinceau 4R. These findings were in agreement with data reported in literature obtained by Raman and chromatographic methods. ${ }^{[6,28]}$ As well as poinceau 4R (1516-, 1436-, 1367-, 1235-, and 1200- $\mathrm{cm}^{-1}$ peaks), additional peaks were found in the spectrum of Giotto Tcolor (1456 and $1281 \mathrm{~cm}^{-1}$ ) attributed to the presence of rhodamine B. Tombow ABT_555 revealed presence of eosin Y (Figure 5b), clearly visible by the overlapping of peaks at 1622, 1504, 1283, 1174, 715, 645 , and $612 \mathrm{~cm}^{-1}$. It should be pointed out that the strong peak at $1080 \mathrm{~cm}^{-1}$ visible in all red pen spectra of Figure 5 belongs to the paper substrate. Regarding the other colors, rhodamine B was identified as main component in pink Carioca Doodles (Figure S3a). Analysis of blue pens (Figure S3b,c) allowed identification of erioglaucine, crystal violet, and rhodamine B in the blue Caran D'Ache ink mixture, whereas erioglaucine and crystal violet were identified in Tombow ABT-555 and Giotto Tcolor pens. Both green pens (Figure S3d) showed diagnostic peaks of erioglaucine and tartrazine, as also found in Stabilo G_68_36.

Finally, the ability of Raman handheld instrumentation for analysis of ink-based modern art was tested with pseudo-real art works. Pink and blue pen lines (from Tombow and Caran D'Ache brands) were traced on paper with felt-tip pens used by Italian artist Federico Fellini and made available by the Foundation Fellini in Cinecitta' (Italy). Figure 6 shows the SERS spectra of the pen lines against the SERS spectra of relevant reference dyes. The pink Tombow SERS spectrum (Figure 6a) showed peaks at 1605,1537 , and $1484 \mathrm{~cm}^{-1}$ that revealed the presence of eosin Y. The blue Caran D'Ache SERS spectrum (Figure 6b) showed peaks attributable to both erioglaucine and crystal violet dyes. Specifically peaks at 1607, 1581, 1176,910 , and $413 \mathrm{~cm}^{-1}$ could be attributed to either dye; peaks at 1535,1386 , and $726 \mathrm{~cm}^{-1}$ were specific to crystal violet. The spectrum appeared different than the other contemporary Caran D'Ache blue examined (Figure S5b). This could be due to the fact that slightly different blues were compared (Caran D'Ache 555 against Fellini Caran D'Ache 100) or that the composition of the blue Caran D'Ache used by Fellini in 1960s has changed with time. The comparison of results obtained with benchtop Raman instrumentation previously carried out in our group ${ }^{[20]}$
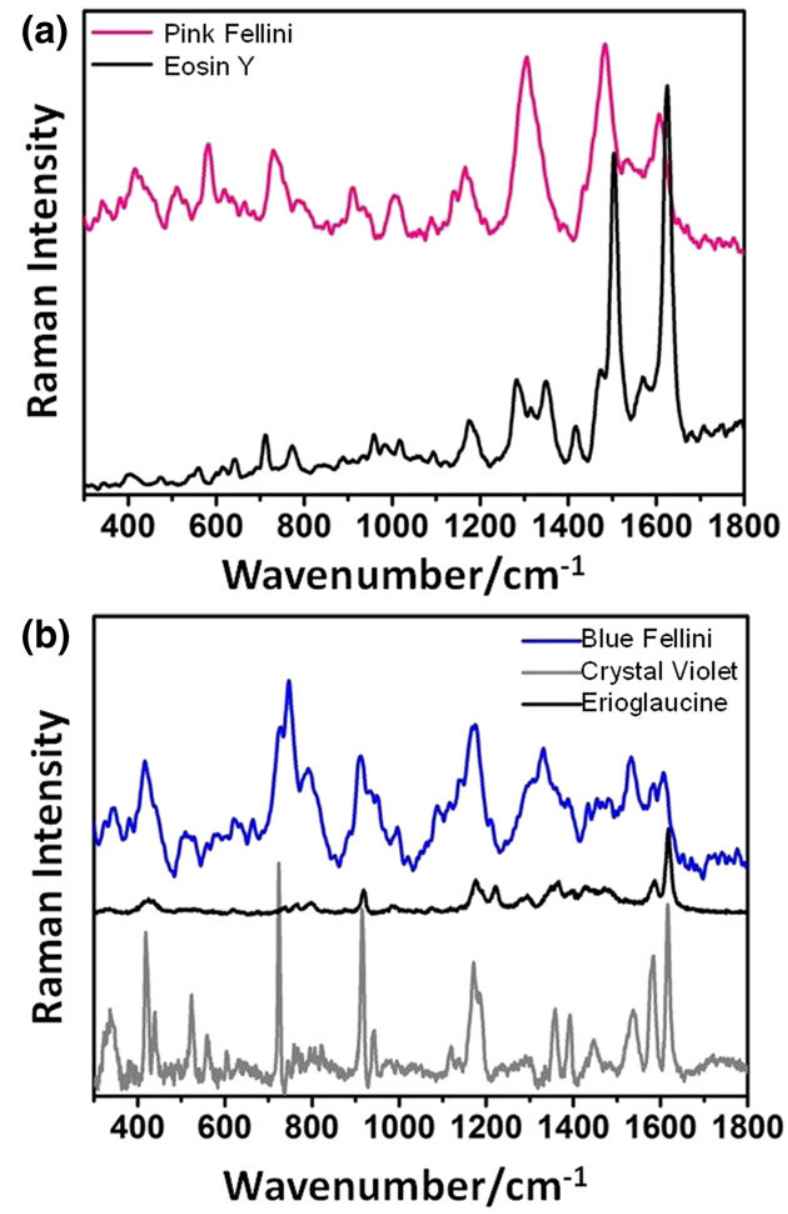

FIGURE 6 Surface-enhanced Raman scattering spectra of colored lines drawn with Federico Fellini's felt-tip pens and surface-enhanced Raman scattering of references: (a) pink Tombow and eosin Y; (b) blue Caran D'Ache, erioglaucine, and crystal violet 
TABLE 2 Summary of all analyzed felt-tip pens and associated identified main dye content

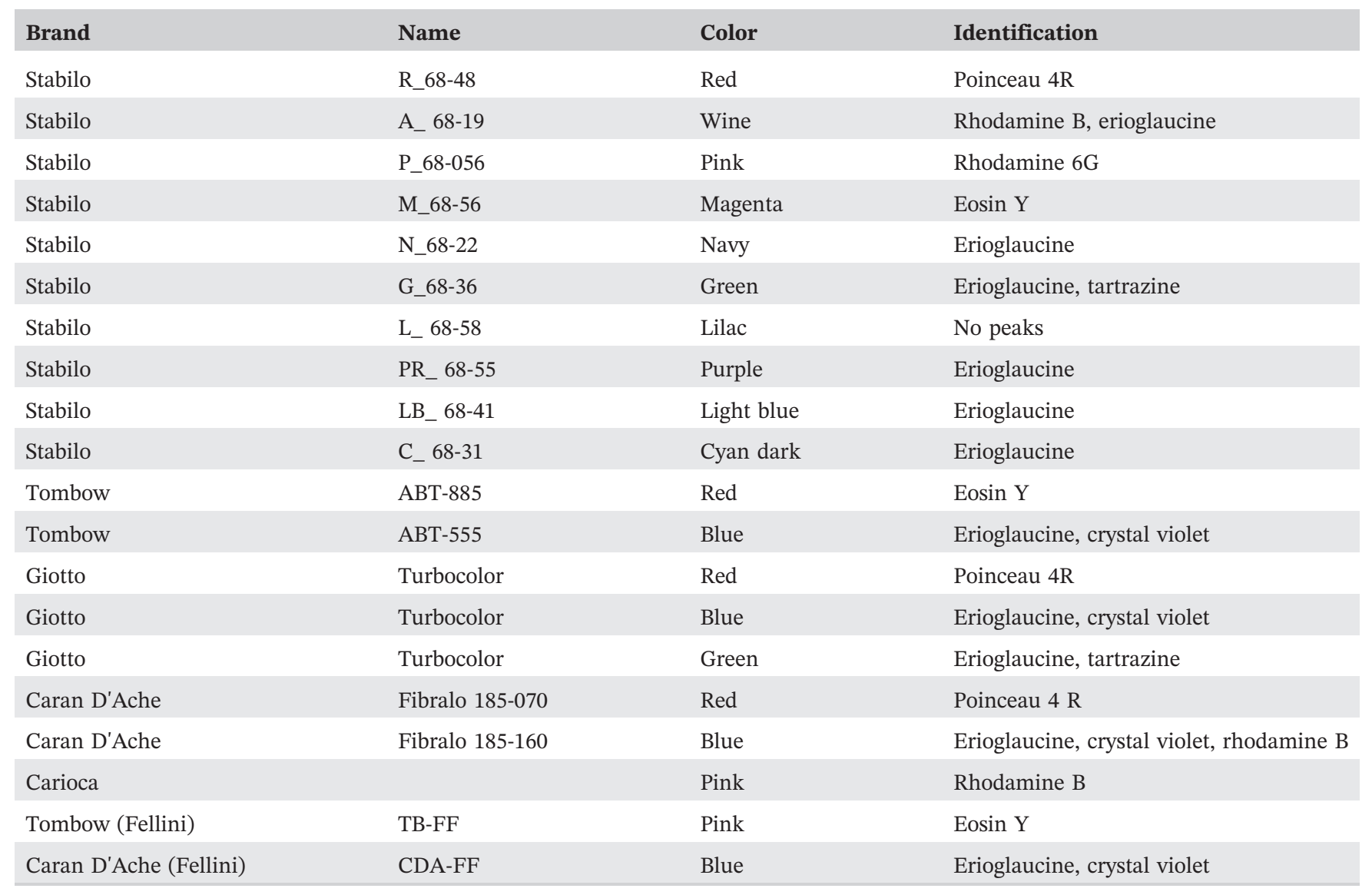

showed that comparable results were obtained under the two experimental conditions (benchtop illumination wavelength $514 \mathrm{~nm}$, resolution $3-5 \mathrm{~cm}^{-1}$, spot size $2-5 \mu \mathrm{m}$, laser power $<2 \mathrm{~mW}$, handheld illumination wavelength $514 \mathrm{~nm}, 10-12 \mathrm{~cm}^{-1}$, resolution, $1 \mathrm{~mm}$ spot size, laser power $50 \mathrm{~mW}$ ). In blue Fellini, additional traces of rhodamine were detected by benchtop Raman illumination. This could be ascribed to a combination of instrumental parameters but also to the different illumination conditions used. In fact, rhodamine's molecular absorbance is very close to the benchtop Raman illumination of $514 \mathrm{~nm}$, leading to high signals due to surface-enhanced resonance Raman scattering (SERRS) conditions. In contrast, the Raman/SERS response of rhodamine at 785-nm illumination is very low.

The summary of all analyzed felt-tip pens and the dye composition attribution is presented in Table 2. Interestingly, some of the pens analyzed in this work were also previously analyzed by a combined bench-size SERS/UV vis spectroscopic approach. ${ }^{[18]}$ The direct comparison between spectra recorded at 514-nm illumination with benchtop instrumentation and spectra recorded in this work at $785-\mathrm{nm}$ illumination with handheld instrumentation showed that sharper peaks with better signal-to-noise ratios were obtained with benchtop instrumentation. Nevertheless, in general, comparable results were obtained with the two analysis. However, due to the higher sensitivity of bench-size instrumentation, the use of SERRS conditions and the combined use of UV-vis spectroscopy, some dye components probably present in small percentages were revealed that could not be detected by handheld measurements. Specifically, rhodamine could not be detected in magenta Stabilo M_68-22, red Tombow and Caran D'Ache, and blue Giotto Tcolor; amaranth and poinceau $4 \mathrm{R}$ could not be detected in red Giotto Tcolor and pink Carioca, respectively.

\section{3 | CONCLUSION}

In conclusion, this work constitutes the first example of characterization of modern ink dye composition by Raman handheld instrumentation. A total of 18 pens of different colors from the commercial brands Stabilo, Caran D'Ache, Giotto, Carioca, and Tombow were analyzed. Red colors could be analyzed by simple NR, but for all remaining colors, the strong interference fluorescence caused by the dyes required the use of SERS. Red colors mainly showed presence of poinceau $4 \mathrm{R}$ dye, and 
pink colors were constituted either by rhodamine B or eosin Y dyes. Blue colors in general showed presence of erioglaucine and crystal violet although some traces of red dyes were also found in some brands. Green colors were always a mix of blue (erioglaucine) and yellow (tartrazine) dyes. The wider applicability of the method was proven by its successful application to different commercial brands and to the analysis of historical pens used by the Italian film director Federico Fellini.

Considering the paramount importance in development of sensitive, cost effective, and deployable instrumentation, this work constitutes an important step beyond the state of the art in the field of diagnostics for modern art conservation. The deposition of nanoinks as SERS probes on works of art is still prohibited by the inherent invasive nature of the process. Nevertheless, this work considerably expands the applicability and versatility of NR and SERS in heritage science and opens up possibilities for the future analysis in situ of large and/or irregularly shaped and/or immovable works of art.

\section{4 | EXPERIMENTAL SECTION}

\section{1 | Materials}

Silver nitrate, sodium citrate, and $\mathrm{MeOH}$ were purchased from Sigma-Aldrich. Reference dyes rhodamine B, rhodamine $6 \mathrm{G}$, eosin $\mathrm{Y}$, poinceau 4R, crystal violet, erioglaucine, and tartrazine were also purchased from Sigma-Aldrich and used without modification. All glasswares were cleaned with aqua regia prior to Ag nanoink synthesis. Milli-Q water (resistivity $>18 \mathrm{M} \Omega \mathrm{cm}-1$ ) was used throughout the experiments.

\section{2 | Synthesis}

Ag nanoinks were synthesized by a modification of the Lee and Meisel method reported by Polavarapu et al. ${ }^{[21]}$

\section{3 | Characterization}

UV-vis spectra of Ag nanoinks were acquired using an Agilent/HP 8453 UV-vis Spectrophotometer $(200 \mathrm{~nm}<\lambda<1100 \mathrm{~nm})$. SEM images of nanoinks deposited on $\mathrm{SiO} 2$ substrates were acquired using a field emission SEM (JSM-6700F, JEOL UK Ltd.) operating at beam voltages of $2 \mathrm{kV}$. Raman spectra at $785 \mathrm{~nm}$ were obtained from a handheld PharmaID spectrometer. The laser power was around $50 \mathrm{~mW}$, and typical acquisition time was between 0.2 and $10 \mathrm{~s}$. To obtain SERS spectra, the POP method developed by Polavarapu et al. was used. ${ }^{[21]}$ Briefly, the cartridge of a commercial fountain pen was filled with Ag nanoink suspension and used to "write" a
SERS-active square $\left(0.5 \mathrm{~mm}^{2}\right)$ on felt-tip pen colored paper. The nanoink was left to dry overnight in the dark prior SERS analysis.

\section{ACKNOWLEDGEMENTS}

This work was supported by the European H2020 Framework Programme (project 646063 Nanorestart and the Irish Research Council (IRC) grant GOIPD/2015/716. The authors wish to thank the director of the Studio Fellini in Cinecittà, Rome (Italy), Roberto Mannoni, close friend and assistant of Federico Fellini, for authorizing the use of historical felt-tip pens.

\section{ORCID}

Rodorico Giorgi (D) http://orcid.org/0000-0002-7752-4107 Antonio Mirabile (D) http://orcid.org/0000-0001-8676-1828 Daniela Iacopino (D) http://orcid.org/0000-0003-2301-9401

\section{REFERENCES}

[1] C. Morales-Merino, S. Röhrs, F. Meyer, S. Marten, I. Reiche, Kupferstichkabinett Berlin 2016, 24, 89.

[2] G. Pfingstag, J. Soc. Dyers Colour. 1993, 109, 188.

[3] S. Bioletti, R. Leahy, J. Fields, B. Meehan, W. Blau, J. Raman Spectrosc. 2009, 40, 1043.

[4] J. Zięba-Palus, M. Kunicki, For. Sci. Intern. 2006, 158, 164.

[5] I. Nastova, O. Grupče, B. Minnčeva-Šukarova, S. Turan, M. Yaygingol, V. Ozcatal, V. Martinovska, Z. JakovleskaSpirovska, J. Raman Spectrosc. 2012, 43, 1729.

[6] A. Sodo, M. Bicchieri, M. Guiso, M. A. Ricci, G. Ricci, J. Raman Spectrosc. 2012, 43, 1781.

[7] D. L. Jeanmarie, R. P. Van Duyne, J. Electroanal. Interfacial Electrochem. 1977, 84, 1.

[8] J. Reguera, J. Langer, D. Jiménez de Aberasturi, L. M. LizMarzán, Chem. Soc. Rev. 2017, 46, 3866.

[9] A. Campion, P. Kambhampati, Chem. Soc. Rev. 1998, 27, 241.

[10] F. Casadio, M. Leona, J. R. Lombardi, R. P. Van Duyne, Acc. Chem. Res. 2011, 44, 238.

[11] M. Leona, Proc. Natl. Acad. Sci. U. S. A. 2009, 106, 14757.

[12] F. Pozzi, J. R. Lombardi, S. Bruni, M. Leona, Anal. Chem. 2012, 84, 3751.

[13] A. Alyami, D. Saviello, M. A. P. McAuliffe, A. Mirabile, L. Lewis, D. Iacopino, Phys. Chem. Chem. Phys. 2017, 19, 14652.

[14] I. Geiman, M. Leona, J. R. Lombardi, Forensic Sci. 2009, 54, 947.

[15] R. M. Seifar, J. M. Verheul, F. Ariese, U. A. T. Brinkman, C. Gooijer, Analyst 2001, 126, 1418.

[16] P. C. White, Sci. Justice 2003, 43, 149.

[17] G. Germinario, S. Garrappa, V. D'Ambrosio, I. D. van der Werf, L. Sabbatini, Anal. Bioanal. Chem. 2017, 410, 1079. 
[18] D. Saviello, M. Trabace, A. Alyami, A. Mirabile, R. Giorgi, P. Baglioni, D. Iacopino, Talanta 2018, 181, 448.

[19] C. Zaffino, A. Passaretti, G. Poldi, M. Fratelli, A. Tibiletti, R. Bestetti, I. Saccani, V. Guglielmi, S. Bruni, J. Cult. Herit. 2017, $23,87$.

[20] D. Saviello, A. Alyami, M. Trabace, A. Mirabile, R. Giorgi, P. Baglioni, D. Iacopino, RSC Adv. 2018, 8, 8365.

[21] N. G. Greeneltech, A. S. Davis, N. A. Valley, F. Casadio, G. C. Schatz, R. P. Van Duyne, N. C. Shah, J. Phys. Chem. A 2012, $116,11863$.

[22] S. Bruni, V. Guglielmi, F. Pozzi, J. Raman Spectrosc. 2011, 42, 1267.

[23] L. Polavarapu, A. La Porta, S. N. Novikov, M. CoronadoPurchau, L. M. Liz-Marzán, Small 2014, 10(15), 3065.

[24] S. Lin, W.-L. J. Hasi, S. Han, X. T. Lou, F. Yang, D.-Y. Lin, Z.-W. Lu, Anal. Methods 2015, 7, 5289.

[25] I. Persaud, W. E. L. Grossman, J. Raman Spectrosc. 1993, 24, 107.

[26] A. V. Whitney, R. P. Van Duyne, F. Casadio, J. Raman Spectrosc. 2006, 37, 993.
[27] N. Peica, I. S. PavelCîntă Pînzaru, K. V. Rastogi, W. Kiefer, J. Raman Spectrosc. 2005, 36, 657.

[28] F. C. Izzo, C. Vitale, C. Fabbro, H. Van Keulen, Microchem. J. 2016, 124, 919.

\section{SUPPORTING INFORMATION}

Additional supporting information may be found online in the Supporting Information section at the end of the article.

How to cite this article: Saviello D, Di Gioia A, Turenne P-I, et al. Handheld surface-enhanced Raman scattering identification of dye chemical composition in felt-tip pen drawings. J Raman Spectrosc. 2018;1-10. https://doi.org/10.1002/ jrs.5411 